\title{
A Demanda de Medição de Vazão e Volumes de Esgotos em Estações de Tratamento de Esgotos do Interior de Pernambuco
}

Title: The demand of flowmeters and wastewater volumes in sewage treatment plants in the country side of Pernambuco State

\author{
Alexandre José Gomes Moreira \\ Escola Politécnica de Pernambuco \\ Universidade de Pernambuco \\ 50.720-001 - Recife, Brasil \\ alexjgm@ig.com.br
}

\author{
Ana de Fátima Braga Barbosa \\ Escola Politécnica de Pernambuco \\ Universidade de Pernambuco \\ 50.720-001 - Recife, Brasil \\ afbb.direito@yahoo.com.br
}

Resumo
Este artigo é o resultado de um estudo de caso que tem como objetivo apresentar a demanda de
macromedidores para macromedição das Estações de Tratamento de Efluentes (ETEs) do inte-
rior do estado de Pernambuco, indicando, além das quantidades, o custo estimado para a aqui-
sição e instalação dos equipamentos. Apresenta também, uma análise SWOT do programa para
instalação dos macromedidores, mostrando as vantagens e desvantagens, e o porquê da sua
realização.

Palavras-Chave: Macromedidor, esgoto, tecnologia, saneamento, vazão, volume

\begin{abstract}
This article is the result of a case study that aims to present the flowmeters demand for measurement of Sewage Treatment Plants (STPS) in the state of Pernambuco, indicating, in addition to the amounts, the estimated cost for the acquisition and installation of equipment. It also presents a SWOT analysis of the program for installation of flowmeters, showing the advantages and disadvantages, and the reason why.
\end{abstract}

Keywords: Flowmeter, sewage, technology, sanitation, flow rate, volume 


\section{Introdução}

A carência de equipamentos para medição de vazão e volumes em ETEs do interior de Pernambuco é uma realidade.

"Implantar sistemas de medição, tem um papel essencial para às companhias públicas de abastecimento de água, pois proporciona o conhecimento das variáveis envolvi-das no processo (...)". [1]

"A macromedição é o conjunto de medições realizadas nos sistemas de abastecimento de água, desde a captação de água bruta até as extremidades da rede de distribuição. [1]

As variáveis de interesse são: vazão e volume, que podem ser medidas através de macromedidores. A NBR 10977-1989 define vazão como "a quantidade de fluido que escoa através da seção transversal de um conduto por unidade de tempo" [2].

Este estudo visa indicar a demanda de medidores de vazão necessários para a medição das vazões e volumes tratados de esgotos nas ETEs operadas pela Compesa, localizadas basicamente no interior do estado de Pernambuco, bem como, fornecer dados para a aquisição e instalação dos medidores adequados a realidade de cada ETE. Fornecer, também, uma estimativa de custos para aquisição e instalação física dos medidores. Apresentar uma análise indicando as vantagens e desvantagens da implantação de um programa visando a instalação dos medidores.

\section{Objetivo Geral}

Levantar a demanda de macromedidores para as estações de tratamento de esgotos do interior de Pernambuco.

\section{Objetivos Específicos}

- Identificar as ETEs em operação no interior do estado de Pernambuco.

- Quantificar os macromedidores necessários para suprir a demanda de macromedição das ETEs do interior.

- Fornecer estimativa de custos para aquisição de macromedidores e data-logers.

- Fornecer estimativa de custos para os serviços de instalação dos macromedidores e obras civis para caixas de proteção dos equipamentos.

- Apresentar análise indicando as vantagens e desvantagens da implantação de um programa para macromedição das ETEs do interior.

\section{Metodologia}

O estudo consistiu em identificar, com base em documentos internos da Compesa, as ETEs em operação no interior do estado.

Após a identificação, foram realizadas visitas a cada ETE, para verificar a realidade local e levantar informações para subsidiar a escolha da tecnologia de medição mais adequada à realidade. Foi elaborado check-list, para padronizar a coleta de dados, onde foram verificadas informações, tais como: presença de operador na unidade, risco de depredação, existência de calha parshall na chegada da unidade, se o sistema possui Estação Elevatória de Esgotos (EEE), e se havia condições de instalação de macromedidor na saída da EEE.

\section{Macromedidores}

Os macromedidores são equipamentos individuais, res-ponsáveis por realizar a macromedição de fluidos. Hoje, medidores de vazão já vem sendo utilizados para medi-ções de esgoto [3]. A instalação de macromedidores, nas ETEs operadas pela Compesa, propiciará o conhecimen-to da vazão e dos volumes produzidos, subsidiará o con-trole operacional das estações, propiciando o atendimento a legislação, especificamente a resolução CONAMA 430/2011 [4].

\subsection{A Macromedição E A Resolu-Cão Co- nama 430/2011}

A Resolução CONAMA 430/2011 dispõe sobre condições, parâmetros, padrões e diretrizes para gestão do lançamento de efluentes em corpos de água receptores. Basicamente, estabelece os limites para parâmetros físicos-químicos que os efluentes devem possuir, para serem lançados em corpos de água. Em termos de volumes, não estabelece valores, porém, estão estabelecidos limites de concentração de poluentes que o efluente deve obedecer. Conhecendo com a maior precisão possível a quantidade de efluente que lança nos corpos receptores, os responsáveis poderão mensurar com maior confiança, os parâmetros exigidos pela resolução em seus lançamentos. Segundo o art. 24 da referida resolução, "os responsáveis pelas fontes poluidoras dos recursos hídricos deverão realizar o automonitoramento para controle e acompanhamento periódico dos efluentes lançados nos corpos receptores, com base em amostragem representativa dos mesmos." Extrapolando a ideia que o texto do artigo nos passa, acreditamos na necessidade de monitoramento das vazões e volumes tratados e lançados nos corpos de água, para um efetivo controle operacional.

\subsection{Classificação dos Medidores}


A NBR 10396-1988 [5] classifica os medidores em 2 grandes grupos: volumétricos e vazão instantânea.

\subsubsection{Medidores volumétricos}

Medidor no qual o volume do fluido é determinado pelo enchimento e esvaziamento alternados de câmaras de capacidade determinada. São exemplos de medidores volumétricos: diafragma, pistão, rotor, palheta, multirotor, disco de nutação.

\subsubsection{Vazão instantânea}

Medidor no qual o fluido passa através do elemento primário em escoamento contínuo, fornecendo uma indicação instantânea proporcional à variação da vazão.

Definições: A NBR 10977-1989 [2] define elemento primário e ele-mento secundário em medição de vazão.

Elemento primário: dispositivo que recebe um estímulo diretamente do fluido que escoa em um determinado conduto e produz um sinal que permite a determinação de vazão. De acordo com o princípio utilizado, o elemento primário pode ser interno ou externo ao conduto.

Elemento secundário: Dispositivo que, recebendo o sinal do elemento primário, mostra, registra, transforma ou transmite a fim de obter o valor da vazão.

A Figura 1 ilustra a classificação dos macromedidores, conforme a NBR 10396-1988 [5].

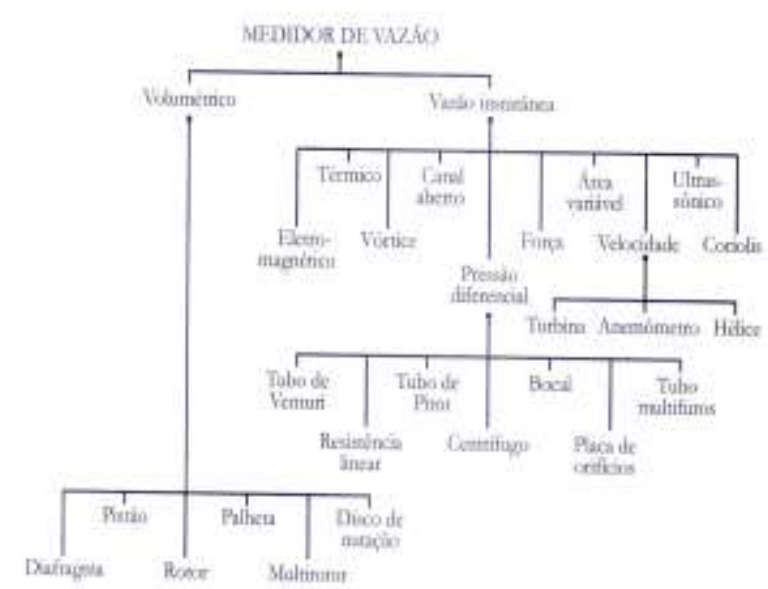

Figura 1: Classificação dos medidores de vazão. Fonte: ABNT NBR 10396-1988.

\subsection{Tipos de macromedidores que po- dem ser aplicados em medições de esgotos}

Nem todos os medidores apresentados se aplicam para medição de esgotos. Os medidores volumétricos citados na NBR 10396-1988 não são utilizados para medição de vazão em esgotos, pois todos apresentam partes móveis, que se deslocam em função de um volume fixo de fluido. Não são recomendados para fluidos que contenham partí- culas em suspensão, como é o caso de esgotos. Pelo mesmo motivo também não são utilizados para medição de vazão em esgotos os medidores de velocidade. Os medidores de pressão diferencial exigem a passagem de fluidos por orifícios muitas vezes de pequeno diâmetro, e não são utilizados para esgotos pois podem sofrer obstrução que causariam grandes erros nas medições. Medidores térmicos são usados para gases e não são utilizados em saneamento. Medidores vórtice possuem orifícios para transmitir pressão, que podem obstruir e não são utilizados para medições de esgoto.

Podemos listar como medidores de vazão que podem ser utilizados para medição de esgotos:

\subsubsection{Vertedouros e calhas}

São medidores para canal aberto, instrumentos tradicionais na medição de vazão, seu funcionamento se baseia na diferença do nível de água que se estabelece quando da interposição de um estrangulamento de seção ou colocação de um obstáculo de fundo no escoamento a lâmina livre. Dentre os medidores de canal aberto, o mais utilizado no saneamento é, sem dúvida, a calha Parshall [6].

\section{Vantagens:}

- Não necessita de fornecimento externo de energia;

- São equipamentos de baixo custo;

- Não necessita de manutenção frequente, limitandose à limpeza de seus canais;

- Permite a medição de líquidos com sólidos em suspensão;

\section{Desvantagens}

- Necessita da existência de canais de entrada nas Estações de Tratamento;

- Baixa resolução da medição.

\subsubsection{Medidores Eletrônicos}

\section{i. Medidores de vazão (nível eletrônico)}

Estes podem ser instalados nos vertedouros e calhas, possibilitando a medição, indicação e totalização dos volumes. São basicamente, medidores de nível ultrassônicos, onde a velocidade do som é a base para a medição, através da técnica de eco. As ondas de ultrassom são geradas por materiais que são excitados em um transdutor, deslocam-se indo de encontro ao fluido, são refletidas por ele, e a distância percorrida pelas ondas é calculada, com base na velocidade de propagação das ondas e do tempo de retorno ao transdutor. 


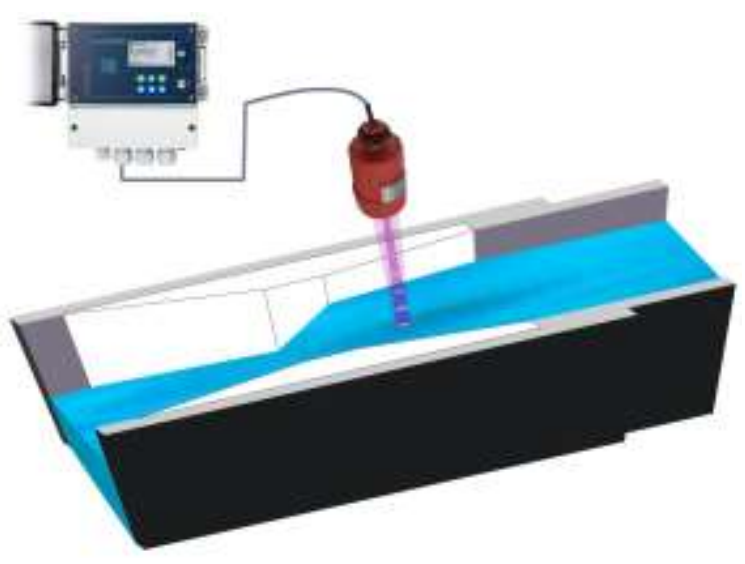

Figura 2: Calha Parshal com medidor de vazão eletrônico - Fonte: www.nivetec.com.br

\section{Vantagens:}

Baixo custo de aquisição, exige pouca manutenção.

\section{Desvantagens:}

Necessita de fonte de energia próxima.

\section{ii. Medidores eletromagnéticos}

Estes são medidores que se baseiam na lei de Faraday de indução eletromagnética para realizar a medição. Esta lei diz que se um fluido condutor cruza perpendicularmente um campo magnético, uma tensão é gerada entre 2 eletrodos, e essa tensão será proporcional à velocidade do fluido. São medidores utilizados em condutos fechados. Exigem para um perfeito funcionamento que toda a seção interna do medidor esteja completamente preenchida pelo líquido a medir, ou seja, que o conduto esteja sob pressão. Contudo, existe um modelo de medidor eletromagnético capaz de medir tubulações parcialmente preenchidas.

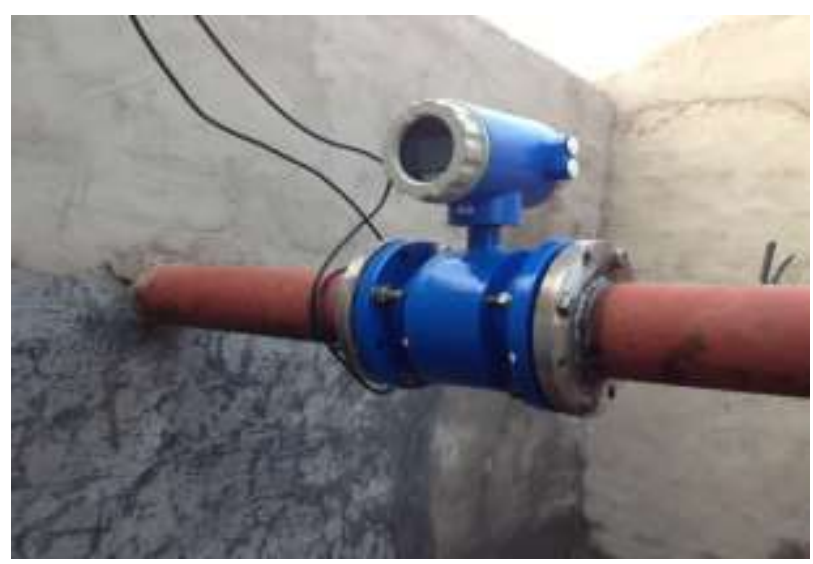

Figura 3: medidor de vazão eletromagnético versão compacta instalado - Fonte: https://sanesoluti.com.br/

\begin{tabular}{|l|l|}
\hline Vantagens & Desvantagens \\
\hline $\begin{array}{l}\text { Apresenta grande preci- } \\
\text { são. }\end{array}$ & $\begin{array}{l}\text { Custo de aquisição e ins- } \\
\text { talação altos. }\end{array}$ \\
\hline $\begin{array}{l}\text { Não possui peças móveis. } \\
\text { Baixa manutenção. }\end{array}$ & $\begin{array}{l}\text { Grande cuidado deve ser } \\
\text { dado à instalação elétrica } \\
\text { e aterramento do medidor. }\end{array}$ \\
\hline $\begin{array}{l}\text { Virtualmente insensível à } \\
\text { densidade e à viscosidade } \\
\text { do fluido de medição. }\end{array}$ & $\begin{array}{l}\text { Necessita de fonte de } \\
\text { energia próxima. }\end{array}$ \\
\hline
\end{tabular}

Tabela 1: Vantagens e desvantagens do medidor eletromagnético carretel - Fonte: o autor.

\section{iii. Medidores ultrassônicos efeito doppler}

Os medidores ultrassônicos para condutos fechados que são usualmente utilizados para medir esgotos são os do tipo "clampon". Estes são compostos de transdutores (sensores) e unidade eletrônica para indicação da vazão/volumes. Os transdutores são aplicados por fora das tubulações, exigindo para isso a aplicação de gel, que propicia a propagação do som. Faz-se necessário o conhecimento da espessura da parede da tubulação, para a correta medição com esses medidores.

\begin{tabular}{|l|l|}
\hline Vantagens & Desvantagens \\
\hline Fácil instalação. & Alto custo \\
\hline Boa precisão & $\begin{array}{l}\text { Requer reposição de gel } \\
\text { do transdutor com certa } \\
\text { frequência }\end{array}$ \\
\hline- & $\begin{array}{l}\text { Incrustações na tubula- } \\
\text { ção causam medições } \\
\text { imprecisas }\end{array}$ \\
\hline
\end{tabular}

Tabela 2: Vantagens e desvantagens do medidor ultrassônico clampon - Fonte: o autor.

\section{As ETEs operadas pela COMPESA}

Após o contrato de Parceria Público Privada (PPP) firmado entre a Compesa e a Odebrecht Ambiental, a operacionalização do tratamento de esgotos na RMR e na cidade de Goiana foi concedido à segunda. Cabe à Compesa o tratamento e destinação dos esgotos das cidades do interior do estado e do arquipélago de Fernando de Noronha. São ao todo 35 ETEs em operação, distribuídas conforme a Tabela 3 . 


\begin{tabular}{|l|c|}
\hline \multicolumn{1}{|c|}{ Região } & Qtde. de ETEs \\
\hline Mata Norte & 1 \\
\hline Mata Sul & 6 \\
\hline Agreste & 18 \\
\hline Sertão & 2 \\
\hline Fernando de Noronha & 35 \\
\hline \multicolumn{2}{|c|}{ Total } \\
\hline
\end{tabular}

Tabela 3: Distribuição das ETEs operadas pela Compesa por região do estado de Pernambuco - Fonte: o autor.

Conforme a Tabela 3, a região onde encontramos a maior quantidade de ETES é o sertão, sendo Petrolina a cidade com a maior concentração, contendo 13 ETEs. Número coerente com o tamanho da cidade, e por se encontrar às margens do rio São Francisco, responsável pelo abastecimento da cidade e das outras que encontramse a sua jusante. A Tabela 4 indica as cidades onde existem ETEs no interior e foram realizadas as visitas técnicas.

\subsection{Diagnóstico da macromedição nas ETEs operadas pela Compesa}

Nos sistemas visitados, alguns são compostos por elevatórias que recalcam para as unidades de tratamento. Algumas unidades possuem mais de uma chegada na ETE, e não oferece condições de segurança aos equipamentos para instalação em suas saídas. Foram locados pontos para instalação dos equipamentos, nas saídas de algumas EEE. De um total de 41 macromedidores, hoje temos apenas $4 \mathrm{em}$ funcionamento, que corresponde a $9,75 \%$ do total.

\section{As Tecnologias de tratamento de Es- gotos Predominantes no Estado}

As tecnologias de tratamento de esgotos das ETEs do interior do estado estão distribuídas conforme a tabela 5.

\begin{tabular}{|l|l|}
\hline Tecnologia & Qtde de ETEs \\
\hline Reator UASB + Filtro biológico & 9 \\
\hline Lagoas em série & 18 \\
\hline Reator UASB + lagoas & 7 \\
\hline Reator UASB & 1 \\
\hline Total & 35 \\
\hline
\end{tabular}

Tabela 5: Tecnologias de tratamento predominantes nas ETEs do interior - Fonte: o autor

\begin{tabular}{|l|l|}
\hline Barra de Sirinhaém & operando \\
\hline Rio Formoso & operando \\
\hline Vitória & operando \\
\hline Barreiros & operando \\
\hline Nazaré da Mata & operando \\
\hline Caruaru & operando \\
\hline Batateiras & operando \\
\hline Belém de Maria - loteamento Santa Luzia & operando \\
\hline Jurema & operando \\
\hline Jurema - Laje dos Caroços & operando \\
\hline Jurema - Sítio Salobro & operando \\
\hline Santo Antônio das queimadas & operando \\
\hline Garanhuns & operando \\
\hline Arcoverde - Cohab I & operando \\
\hline Arcoverde - Cohab II & operando \\
\hline Ipubi & operando \\
\hline Petrolina - João de Deus & operando \\
\hline Petrolina - Cohab VI & operando \\
\hline Petrolina - Antônio Cassimiro & operando \\
\hline Petrolina - Ouro Preto & operando \\
\hline Petrolina - Dom Avelar & operando \\
\hline Petrolina - ETE Centro & operando \\
\hline Petrolina - Loteamento Recife & operando \\
\hline Petrolina - Vila Marcela & operando \\
\hline Petrolina - Izacolândia & operando \\
\hline Petrolina - Jardim Petropolis & operando \\
\hline Petrolina - Rajada & operando \\
\hline Lagoa Grande & operando \\
\hline Dormentes & operando \\
\hline Salgueiro & operando \\
\hline Parnamirim & operando \\
\hline F. N Cachorro & operando \\
\hline F.N Boldró & operando \\
\hline ETES Operan & \\
\hline
\end{tabular}

ETES Operando:

35

Tabela 4: Cidades do interior onde existem ETEs e foram realizadas visitas para levantamento da demanda de macromedidores - Fonte: Compesa

\subsection{Síntese das tecnologias}

\subsubsection{Reator UASB}

"O Reator UASB é uma tecnologia de tratamento biológico de esgotos baseada na decomposição anaeróbia da matéria orgânica. Consiste em uma coluna de escoamento ascendente, composta de uma zona de digestão, uma zona de sedimentação, e o dispositivo separador de fases gássólido-líquido. O esgoto aflui ao reator e após ser distribuído pelo seu fundo, segue uma trajetória ascendente, 
desde a sua parte mais baixa, até encontrar a manta de lodo, onde ocorre a mistura, a biodegradação e a digestão anaeróbia do conteúdo orgânico, tendo como subproduto a geração de gases metano, carbônico e sulfídrico. Ainda em escoamento ascendente, e através de passagens definidas pela estrutura dos dispositivos de coleta de gases e de sedimentação, o esgoto alcança a zona de sedimentação" [7].

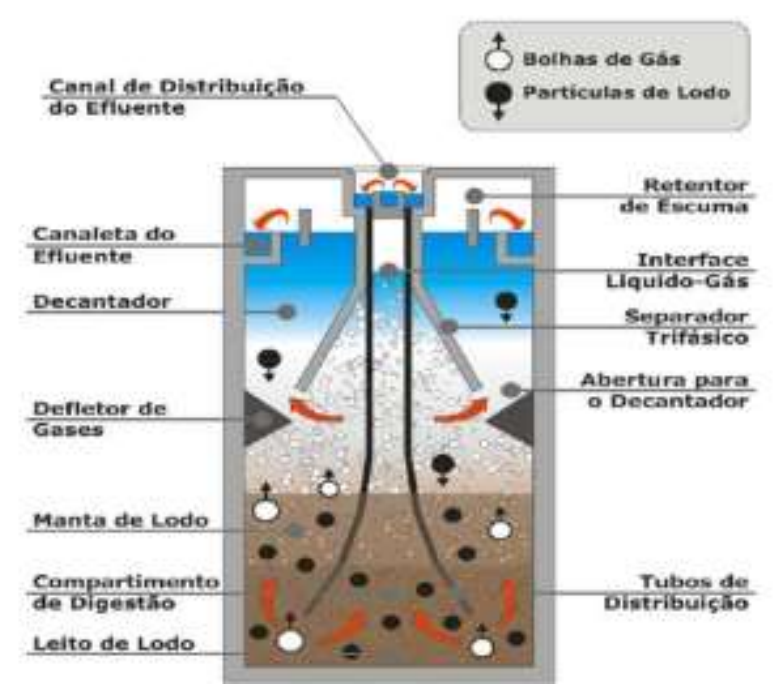

Figura 4: esquema de funcionamento de um reator UASB - - Fonte: http://engenheirosunidos.com.br/estacoes-de-tratamento-de-esgotoreatores-uasb-ou-dafa/

\subsubsection{Filtro Biológico}

"O filtro biológico é constituído de um leito que pode ser de pedras, ripas ou material sintético. O efluente é lançado sobre este por meio de braços rotativos e percola através das pedras (ou outro material) formando sobre estas uma película de bactérias. $\mathrm{O}$ esgoto passa rapidamente pelo leito em direção ao dreno de fundo, porém a película de bactérias absorve uma quantidade de matéria orgânica e faz sua digestão mais lentamente. É considerado um processo aeróbio uma vez que o ar pode circular entre os vazios do material que constitui o leito fornecendo oxigênio para as bactérias. Quando a película de bactérias fica muito espessa, os vazios diminuem de dimensões e a velocidade com que o efluente passa aumenta. Devido a isso surgem forças cisalhantes que fazem com que a película se desgarre do material" [8].

\subsubsection{Lagoas}

"As lagoas de estabilização são consideradas como uma das técnicas mais simples de tratamento de esgotos. Dependendo da área disponível, topografia do terreno e grau de eficiência desejado" [9].
Nesse sistema de tratamento, o esgoto é tratado de forma natural, em lagoas, onde os micro-organismos degradam a matéria orgânica, reduzindo a Demanda Bioquímica de Oxigênio (DBO).

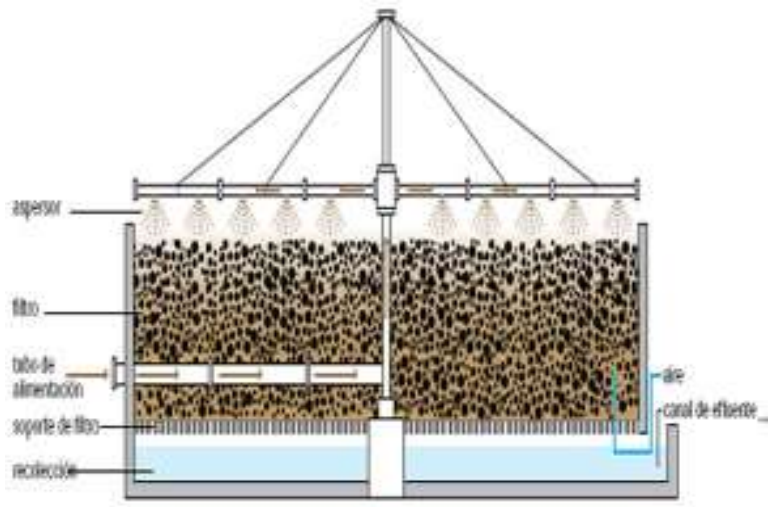

Figura 5: Filtro biológico - Fonte: http://alianzaporelagua.org/Compendio/tecnologias/t/t8.html

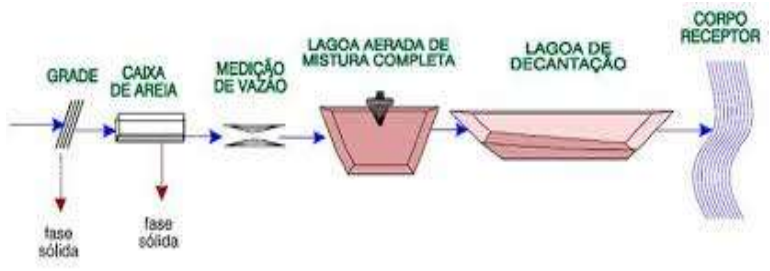

Figura 6: esquema de tratamento por lagoas. - Fonte: http://www.naturaltec.com.br/aeracao-por-difusores.html

\section{Locação de medidores de vazão em função da realidade local}

O estudo em tela foi realizado levando-se em conta a realidade de cada ETE do interior do estado. Foram realizadas visitas in loco para verificar cada realidade.

Segundo Gomes, “(...) deve ser ressaltado que a medição não pode ser vista como uma operação isolada, independente do contexto e das finalidades(...). Não basta dispor de um instrumento e realizar a leitura, há que se estabelecer um contexto onde se dá a medição" [1].

Na locação dos macromedidores foi levado em conta o contexto de cada ETE.

Se havia operador na unidade, a segurança e o risco de depredação do equipamento, se haveria necessidade de construir caixa de proteção para o sensor ou se havia trecho de tubulação aéreo, onde não precisasse construir caixa de proteção (que aumenta custos). 


\section{Tecnologias de medição adotadas sugeridas}

Com base nas realidades encontradas, sugeriu-se 3 tecnologias para medição.

Medidores eletromagnético tipo carretel, versão remota e versão compacta;

Medidores eletromagnético tipo carretel para tubulações com seções parcialmente preenchidas;

Medidores de vazão eletrônicos para calhas e vertedouros.

Os medidores eletromagnéticos tipo carretel de versão compacta foram previstos para instalação nas redes de chegadas de algumas unidades. Nestes medidores os conversores (unidades eletrônicas que apresentam as variáveis ao operador) são acoplados ao sensor. As redes de chegada apresentam trecho aéreo, que facilita a instalação do medidor, bem como o acesso para leitura das variáveis de interesse.

Os medidores eletromagnéticos com indicação remota, foram previstos para instalação na maioria das unidades, e necessitam construção de caixas de proteção, bem como abrigo para os conversores. Os medidores de vazão eletrônicos para calhas e vertedouros, foram previstos para instalação em unidades que apresentam calhas ou vertedouros implantados, e oferecem condições seguras, baixo risco de depredação dos equipamentos.

Além da tecnologia para medição, foi prevista a instalação de data-logers, que são armazenadores eletrônicos de dados. Estes são conectados eletricamente aos macromedidores, e recebem um sinal elétrico proporcional a vazão. Registram os valores, e podem enviá-los via $G e-$ neral Packet Radio Services (GPRS) para uma central. O GPRS é um protocolo de transmissão de dados via linhas de telefone celular.

Foram previstos os equipamentos listados abaixo e distribuídos conforme a tabela 6 :

(i) 19 medidores eletromagnéticos carretel versão remota

(ii) 4 medidores eletromagnéticos carretel versão compacta

(iii) 2 eletromagnéticos carretel seção parcial

(iv) 12 ultrassônicos eletrônicos para calhas e vertedouros

(v) 37 data-logers com tecnologia GPRS

\begin{tabular}{|c|c|}
\hline Diâmetro $(\mathbf{m m})$ & Quantidade \\
\hline 75 (Compacto) & 1 \\
\hline 80 & 6 \\
\hline 100 & 2 \\
\hline 150 & 1 \\
\hline 150 (Compacto) & 2 \\
\hline 200 & 4 \\
\hline 200 (Tidalflux & 3 \\
\hline 250 & 2 \\
\hline 300 & 1 \\
\hline 300 (Compacto) & 1 \\
\hline 400 & 12 \\
\hline 500 & 37 \\
\hline Ultrassônico calhas \\
e vertedouros
\end{tabular}

Tabela 6: Demanda de macromedidores por diâmetro Fonte: elaborada pelo autor.

\section{Custo do projeto}

Foi realizado um levantamento de previsão de custos para o projeto. O projeto prevê:

(i) Aquisição de macromedidores;

(ii) Instalação dos macromedidores e obras civis para construção de caixas de proteção;

(iii) Aquisição de data-logers.

O custo total em Reais previsto está apresentado conforme a Tabela 7, e é detalhado nas tabelas 8 e 9 .

\begin{tabular}{|l|r|}
\hline Macromedidores & $738.870,55$ \\
\hline Instalações e obras & $716.730,38$ \\
\hline Aquisição de data logers & $110.075,00$ \\
\hline Custo Total & $1.565 .675,93$ \\
\hline
\end{tabular}

Tabela 9: Custo total previsto - Fonte: o autor. 


\section{Referências}

[1] G. R. Andrews, F. B. Schneider. Concepts and Notations for Concurrent Programming. Computing Surveys. 15(2):3-43, 1983.

[2] R. A. Brooks. Autonomous Mobile Robots. In W. E. L. Grimson, , R. S. Paul (eds.) AI In The 1990's And Beyond, páginas 343-363, MIT Press, Massachusetts. 1987.

[3] R. A. Brooks. A Robust Layered Control System for a Mobile Robot. AI Memo 864. Massachusetts Institute of Technology, 1985.

[4] E. Gat. ALFA: A Language for Programming Reactive Control Systems. In Proceedings of the IEEE International Conference on Robotics and Automation. páginas 1116-1121, 1991.

[5] C. Hewitt. Viewing Control Structures as Patterns of Passing Messages. Artificial Intelligence. 8: 323-364, 1977.

[6] S. Matsuoka, A. Yonezawa. Analysis of Inheritance Anomaly in Object-Oriented Concurrent Programming Languages. In Research Directions in Concurrent Object Oriented Programming. MIT Press. páginas 107-150, 1993.

[7] H. A. Schneebeli. Die Steuerung von Mehrfinger-Greifersystemen. Universidade de Karlsruhe, Alemanha, Março 1992.

[8] J. E. M. Xavier. Uma Estrutura para a Construção de Sistemas de Controle Baseados em Agentes para Robôs Móveis. Dissertação de Mestrado, Universidade Federal do Espírito Santo, Novembro 1996.

[9] A. Yonezawa, E. Shibayama, T. Takada et al. Modeling and Programming in an ObjectOriented Concurrent Language. In A. Yonezawa, M. Tokoro, (eds.) Object-Oriented Concurrent Programming. MIT Press. páginas 55-90, 1991. 